Results $11 \% \quad(n=16)$ patients presented between 00:00 and 06:00 h, 36\% ( $n=50)$ between 06:00 and 12:00 hours, 30\% $(n=43)$ between 12:00 and 18:00, and $22 \%(n=31)$ between 18:00 and $24: 00\left(\chi^{2}=18.3, p<0.05\right)$. Average Wells Scores were $4(S D \pm 2), 6$ $(\mathrm{SD} \pm 1), 4(\mathrm{SD} \pm 2)$ and $5(\mathrm{SD} \pm 2)$ for the respective times. Patients with bilateral emboli affecting the main pulmonary arteries were distributed as follows: 00:00-06:00 $(\mathrm{n}=2)$, 06:00-12:00 ( $n=17), 12: 00-18: 00(n=10), 18: 00-24: 00 \quad(n=9)\left(\chi^{2}\right.$ $=10.7, \mathrm{p}<0.05)$. Patients with unilateral emboli affecting the main pulmonary arteries were found to present as follows: 00:00-06:00 ( $n=3), 06: 00-12: 00 \quad(n=11), 12: 00-18: 00 \quad(n=10)$, 18:00-24:00 $(n=7)\left(\chi^{2}=5.00, p=0.17\right)$. Patients with bilateral emboli affecting the segmental arteries presented at: 00:00-06:00 $(n=4), 06: 00-12: 00 \quad(n=12), 12: 00-18: 00 \quad(n=8), 18: 00-24: 00$ $(\mathrm{n}=3) \quad\left(\chi^{2}=7.52, \quad \mathrm{p}=0.05\right)$. Patients with unilateral emboli affecting the segmental arteries presented at: 00:00-06:00 $(\mathrm{n}=5), \quad 06: 00-12: 00 \quad(\mathrm{n}=5), \quad 12: 00-18: 00(\mathrm{n}=8), \quad 18: 00-24: 00$ $(n=5) .\left(\chi^{2}=1.17, p=0.76\right)$ In the 4 time intervals, patients with $>2$ symptoms of chest pain, dyspnoea, or haemoptysis were found to be distributed as: 00:00-06:00 ( $n=8), 06: 00-12: 00$ $(\mathrm{n}=26), 12: 00-18: 00 \quad(\mathrm{n}=16), 18: 00-24: 00 \quad(\mathrm{n}=17) \quad\left(\chi^{2}=9.72\right.$, $\mathrm{p}<0.05)$.

Conclusion Pulmonary Emboli were most frequent between 06:00 and $12: 00 \mathrm{~h}$ during which there was more extensive radiographical findings, associated with a higher Wells score, and more profound symptoms. This suggests a circadian pattern of the presentation of pulmonary emboli, correlating with the clinical and radiological severity of disease.

\section{P256 INVESTIGATING SUSPECTED PULMONARY EMBOLISM AS OUTPATIENT: THE PORTSMOUTH EXPERIENCE}

doi:10.1136/thx.2010.151076.7

I Shafiq, N Siddique, A J Chauhan, L Albon, V King, S R Alapati. Queen Aexandra Hospital, Portsmouth, UK

Introduction Suspected PE is a commonacute medical presentation. It continues to be a significant burden on the healthcare resources, hence it makes sense to investigate and manage stable patients with suspected PE as outpatient. There is very limited evidence available on the safety of investigation of PE as outpatient. The aim of this study was to review the outcomes of investigation for PE on outpatient basis.

Methods During 6 months period from November 2007 to April 2008, 176 patients were investigated for suspected pulmonary embolism as outpatient, based on clinical criteria of stability (eg notachycardia, tachypnea or hypotension). All of these patients were treated withenoxaparin from the day of admission till the diagnosis of PE was confirmed or excluded. We randomly selected 92 of these patients and retrospectively reviewed their clinical notes. The data recorded included pulse, blood pressure, respiratory rate, $\mathrm{PO}_{2}, \mathrm{PCO}_{2}$, Trop t and D.dimer. We also looked at the number of days patients had to wait for the CTPA or $\mathrm{VQ}$ scan. All patients were reviewed in clinic after a VQ scan or CTPA.

Results 12 out of 91 (13.2\%) patients were diagnosed with PE. There were no deaths and no significant complications recorded from either PE or enoxaparin therapy. Average time taken for $\mathrm{PE}$ to be diagnosed or excluded was 3.86 days. There were no significant differences in clinical or physiological parameters between groups apart from $\mathrm{PO}_{2}$, which was significantly lower in the group with PE (p 0.032) (Abstract P256 Table 1).
Abstract P256 Table 1

\begin{tabular}{lllll}
\hline Characteristic & Total (mean) & PE diagnosed & PE excluded & p-Value \\
\hline $\mathrm{n}$ & 91 & $12(13.2 \%)$ & $79(86.8 \%)$ & \\
Age & 50.01 & 56.83 & 49 & 0.187 \\
Female & 63 & $7(11.1 \%)$ & $56(88.9 \%)$ & \\
$\mathrm{Male}$ & 28 & $5(17.9 \%)$ & $23(82.1 \%)$ & \\
$\mathrm{PO}_{2}(\mathrm{kPa})$ & 10.96 & 9.84 & 11.15 & 0.032 \\
$\mathrm{PCO}_{2}(\mathrm{kPa})$ & 5.28 & 4.66 & 5.38 & 0.214 \\
$\mathrm{RR}(\mathrm{n} / \mathrm{min})$ & 17.42 & 17.58 & 17.4 & 0.844 \\
$\mathrm{SaO}$ & & 96.33 & 97.12 & 0.403 \\
$\mathrm{Systolic}$ & 97.01 & 130.41 & 141.83 & 0.248 \\
Diastolic & 140.32 & 81.16 & 80.59 & 0.909 \\
$\mathrm{HR}$ & 80.67 & 92.25 & 84.1 & 0.166 \\
Trop T & 85.2 & 0.14 & 0.05 & 0.408 \\
D.Dimer & 0.07 & 2.28 & 1.18 & 0.295 \\
Request to test time (days) & 3.86 & 5 & 3.68 & 0.427 \\
\hline
\end{tabular}

Conclusions We conclude from this small series that it may be safe to investigate suspected $\mathrm{PE}$ as outpatient in selected clinically stable patients, though this needs to be confirmed in larger studies with an evaluation of health economic benefits.

\section{P257 A COMPARISON OF SCORING SYSTEMS IN THE MANAGEMENT OF A RANGE OF PULMONARY EMBOLISM PATIENTS IN A UNIVERSITY HOSPITAL}

doi:10.1136/thx.2010.151076.8

M Maruthappu, A Manuel, A Christian, A Hollington, M Ramsden, Z Alexopoulou, M Healy, M Giles. University of Oxford, Oxford, UK

Introduction Pulmonary embolism (PE) is a leading cause for inpatient admission and in patient mortality in the UK. Its clinical features are often nonspecific, making a diagnosis of pulmonary embolism difficult and without appropriate treatment; a pulmonary embolism can be fatal. We compared three scoring systems (Geneva and Wells score, which are both predictive tools and Pulmonary Embolism Severity Index (PESI) a risk stratification tool) in three distinct patient groups; those whose primary cause of death was pulmonary embolism, those whose management required admission or patients managed on an outpatient basis.

Methods A retrospective review of case notes for patients with the primary diagnosis of pulmonary embolism from 2009 to 2010 was performed at the Oxford Radcliffe NHS Trust, applying the Wells, PESI and Geneva scoring systems. Death from PE was defined by the presence of a PE or it being listed as the primary cause of death on the death certification in combination with concordant view of a senior clinician of the medical team. Outpatient management was based on patients having a zero length-of-stay. All groups were distinct.

Results See Abstract P257 Table 1 for selected results.

Discussion Across all our group of patients, the PESI outperformed both the Wells and Geneva score. Patients who died from PE were older and more hypoxic, and often caused most diagnostic difficulty presenting with non-respiratory symptoms in over half of the cases. Abnormal chest radiograms were common in all groups and although Ddimer assists in diagnosis other biomarkers such as troponin and BNP were not helpful. The PESI also outperformed the other scores as aid on deciding to manage patients with PE as an outpatient, but still with a degree of uncertainty.

In conclusion, PESI should be considered in the management and risk stratification of PE and PE should be always considered in older patients with non specific clinical features, abnormal 
Abtsract P257 Table 1 Selected results

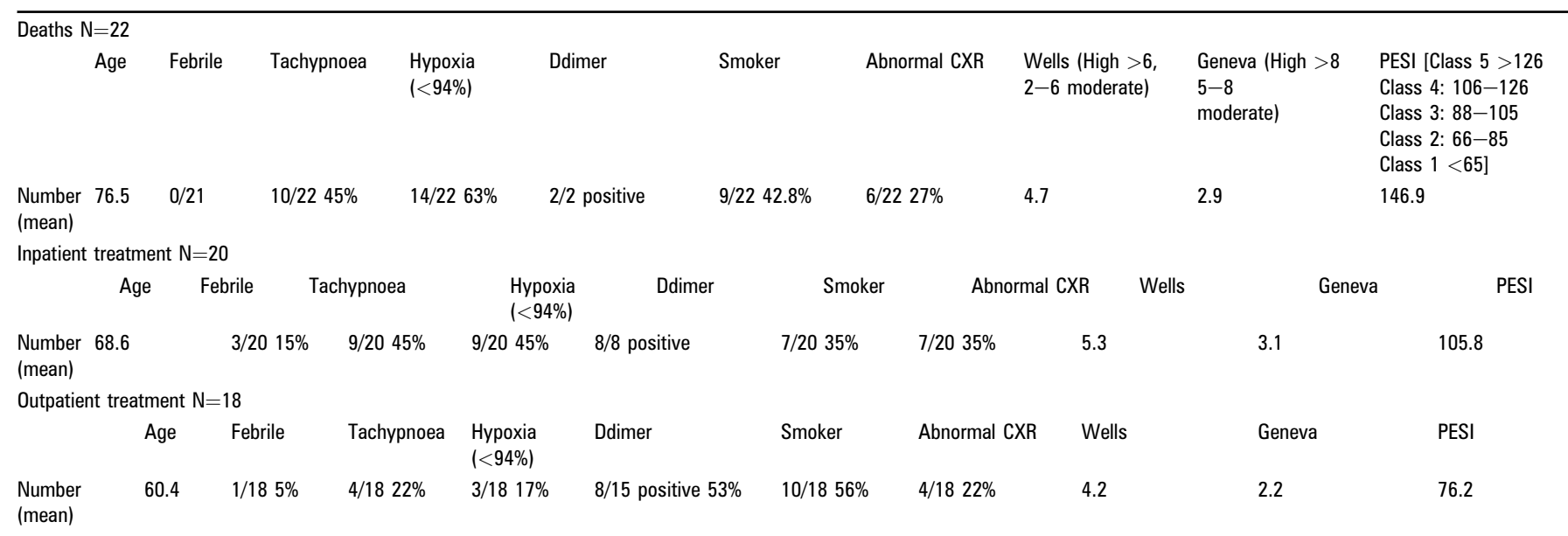

radiology and hypoxia. Managing $\mathrm{PE}$ as an outpatient potentially requires a combination of scoring systems in a prospective study.

\section{P258 IS CHRONIC THROMBOEMBOLIC PULMONARY HYPERTENSION (CTEPH) UNDER DIAGNOSED IN THE UK?}

doi:10.1136/thx.2010.151076.9

${ }^{1} \mathrm{~S}$ Sturney, ${ }^{1} \mathrm{G}$ Robinson, ${ }^{2} \mathrm{G}$ Coghlan, ${ }^{3} \mathrm{~J}$ Pepke-Zaba, ${ }^{1} \mathrm{~J}$ Easaw, ${ }^{1} \mathrm{~J}$ Suntharalingam. ${ }^{1}$ Royal United Hospital, Bath, UK; ${ }^{2}$ Royal Free Hospital, London, UK; ${ }^{3}$ Papworth Hospital, Cambridge, UK

Introduction Chronic Thromboembolic Pulmonary Hypertension $(\mathrm{CTEPH})$ is a rare but treatable condition. In selected cases Pulmonary Endarterectomy (PEA) surgery is potentially curative, with 80-85 procedures performed annually at a single national centre, Papworth Hospital. Previous studies suggest the disease may be under diagnosed.

Aim To identify whether developing a local Pulmonary Hypertension (PH) service improves the number of CTEPH cases diagnosed in a DGH setting.

Methods In 2005, a regional PH satellite service was set up at the Royal United Hospital (RUH), Bath, in conjunction with the Royal Free, London. The service aims to provide local access to PH-specific therapies at national standards of care. New cases of CTEPH are investigated with a number of investigations including right heart catheterisation and pulmonary angiography and then referred to Papworth Hospital's MDT to determine eligibility for surgery. This abstract examines the number of cases diagnosed in RUH's own catchment area following a rolling education programme targeting local physicians and radiologists.

Results Prior to 2007 no RUH patients had undergone PEA surgery. Since 2007, 12 patients have been diagnosed with CTEPH (7 male) with mean age 62.3 years (SD 12.1, range $37-72)$. Only $5(41.7 \%)$ had a documented history of previous thromboembolic disease. Referrals were received from a variety of sources including respiratory, vascular, rheumatology, gastroenterology, primary care, ENT, stroke physician and cardiology. Four (33.3\%) were referred as inpatients. At referral 33.3\% were NYHA class II, $41.7 \%$ class III and $25.0 \%$ class IV. Mean pulmonary haemodynamics were: RA $10.9 \mathrm{~mm} \mathrm{Hg}$ (6.4), mPAP $46.3 \mathrm{~mm} \mathrm{Hg}$ (13.1), PCWP $13.2 \mathrm{~mm} \mathrm{Hg}$ (4.4), CO 4.81/min (0.9), PVR 7.8WU (4.1). 8 have undergone or are awaiting surgery, 1 died of unrelated causes and 3 are being treated medically. (Abstract P258 Figure 1).

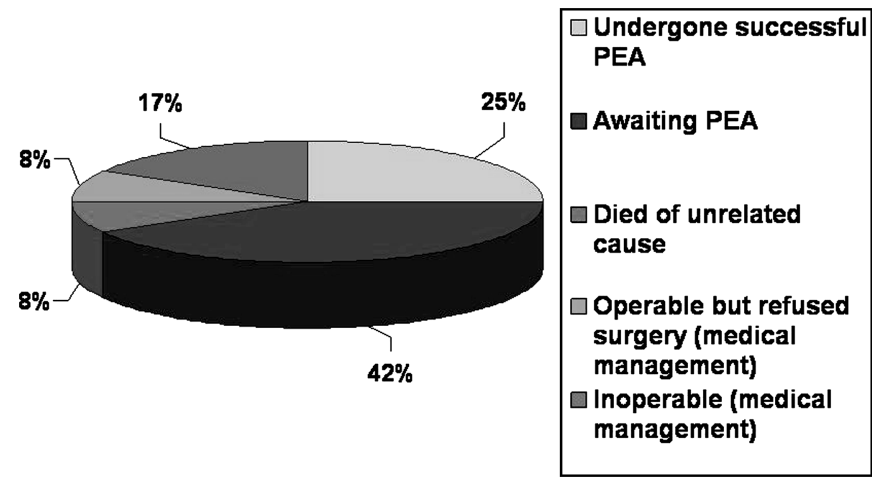

Abstract P258 Figure 1 Outcomes for patients with CTEPH diagnosed at RUH since 2007.

Conclusions Underdiagnosis of CTEPH may be exacerbated by patients presenting to a variety of specialties. The number of CTEPH cases diagnosed at the RUH has increased significantly since 2007, probably as a result of raised local awareness of the condition. It is therefore likely that this potentially curable condition is under diagnosed in areas with more limited access to $\mathrm{PH}$ specialist facilities.

\section{P259 INCIDENTAL FINDINGS ON CT PULMONARY ANGIOGRAPHY: WHAT SHOULD WE BE LOOKING FOR?}

doi:10.1136/thx.2010.151076.10

R Thiagarajah, M Qureshi, A Sahu, R Riordan. Peninsula Radiology Academy, Plymouth, UK

Introduction and Objectives The role of diagnostic imaging in pulmonary embolism (PE) is being increasingly undertaken by CT pulmonary angiography (CTPA) and there has been an explosion of usage in our trust in the last two years, which reflects a growing trend in clinical practice in the United Kingdom (UK). There has been an abundance of literature on how best to utilise CTPA with particular attention to optimisation of pulmonary artery opacification to aid diagnosis. An additional benefit of CTPA over other modalities for the diagnosis of PE that may be less widely appreciated is CTPAs ability to provide simultaneous information on other pathologies affecting the thorax and alternative diagnoses requiring further clinical management.

Methods To this cause a sample of 400 CTPAs were retrospectively reviewed in a 1001 bed acute teaching hospital in the UK, to identify 